LA-UR- $9:=3585$ (final $9 / 17 / 97$

\title{
Title: TRANSIENT X-RAY DIFFRACTION AND ITS APPLICATIONS TO MATERIALS SCIENCE AND X-RAY OPTICS
}

$$
\text { CONF-970706-- }
$$

\begin{tabular}{|c|c|c|}
\hline Author(s): & $\begin{array}{l}\text { A. A. Hauer, P-24 } \\
\text { D, Kalantar, LLNL } \\
\text { R. Kopp, X-PA } \\
\text { B. Failor, Physics International } \\
\text { M. Meyers, UCSD } \\
\text { T. Boehley, Univ. of Rochester L } \\
\text { Energetics }\end{array}$ & $\begin{array}{l}\text { J. S. Wark Univ. of Oxford } \\
\text { B. Remington, LLNL } \\
\text { J. Cobble, P-24 } \\
\text { G. Kyrala, P-24 } \\
\text { R. Springer, MST-7 } \\
\text { aboratory for Laser }\end{array}$ \\
\hline
\end{tabular}

Submitted to:

SPIE CONFERENCE PROCEEDINGS

SAN DIEGO, CA

31 JULY 1997

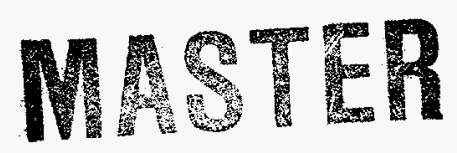

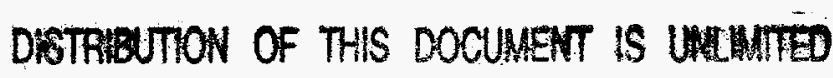

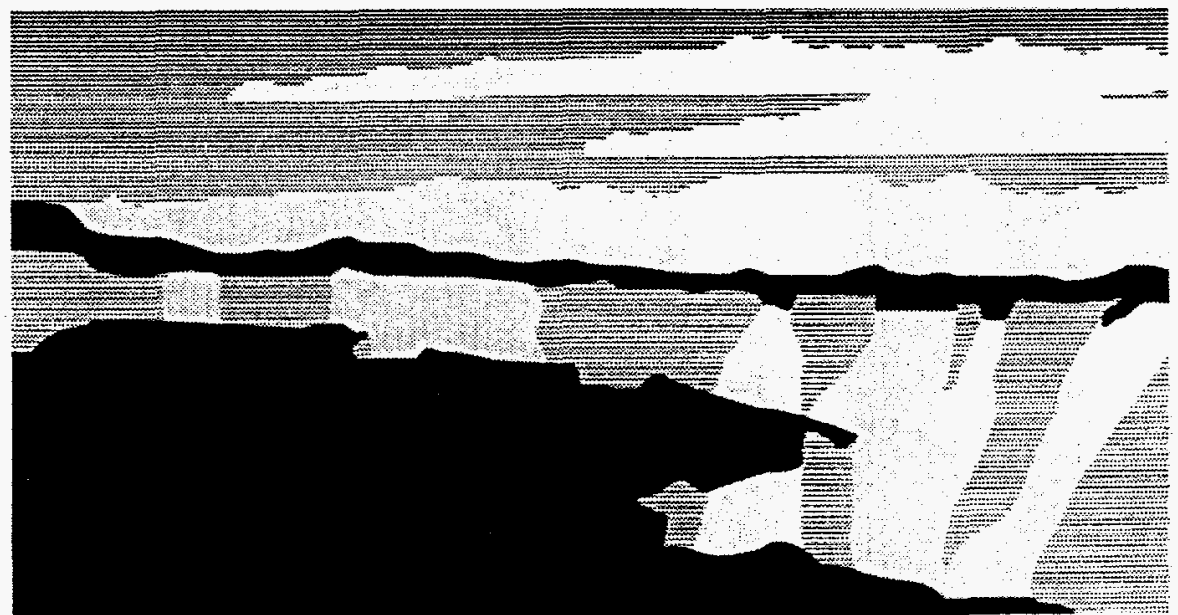

NATIONAL LABORATORY

Los Alamos National Laboratory, an affirmative action/equal opportunity employer, is operated by the University of California for the U.S. Department of Energy under contract W-7405-ENG-36. By acceptance of this article, the publisher recognizes the U.S. Government retains a nonexclusive, royalty-free license to puplish or reporduce the published form of this contribution, or to allow others to do so, for U.S. Government purposes. The Los Alamos Natinal Laboratory requests that the publisher identify this article as work performed under the auspices of the U.S. Department of Energy. 


\section{DISCLAIMER}

This report was prepared as an account of work sponsored by an agency of the United States Government. Neither the United States Government nor any agency thereof, nor any of their employees, makes any warranty, express or implied, or assumes any legal liability or responsibility for the accuracy, completeness, or usefulness of any information, apparatus, product, or process disclosed, or represents that its use would not infringe privately owned rights. Reference herein to any specific commercial product, process, or service by trade name, trademark, manufacturer, or otherwise does not necessarily constitute or imply its endorsement, recommendation, or favoring by the United States Government or any agency thereof. The views and opinions of authors expressed herein do not necessarily state or reflect those of the United States Government or any agency thereof. 


\section{DISCLAMVIXR}

Portions of this doecoment may be illegible in electronic ironge produets. Images are produced from the best available original docoment 


\title{
Transient X-ray Diffraction and Its Application to Materials Science and X-ray Optics
}

\author{
A.A. Hauer ${ }^{1}$, J.S. Wark ${ }^{2}$, D.Kalantar ${ }^{3}$, B. Remington ${ }^{3}$, R. Kopp ${ }^{1}$ J. Cobble ${ }^{1}$, B. Failor ${ }^{4}$, G. Kyrala ${ }^{1}$, \\ M. Meyers ${ }^{5}$, R. Springer ${ }^{1}$, T. Boehley ${ }^{6}$ \\ 1. Los Alamos National Laboratory, 2. University of Oxford, 3. Lawrence Livermore National \\ Laboratory, 4. Physics International, 5. University of California, San Diego,6. University of \\ Rochester, laboratory for LaserEnergetics
}

\begin{abstract}
Time resolved $\mathrm{x}$-ray diffraction and scattering have been applied to the measurement of a wide variety of physical phenomena from chemical reactions to shock wave physics. Interest in this method has heightened in recent years with the advent of versatile, high power, pulsed x-ray sources utilizing laser plasmas, electron beams and other methods. In this article, we will describe some of the fundamentals involved in time resolved x-ray diffraction, review some of the history of its development, and describe some recent progress in the field. In this article we will emphasize the use of laser-plasmas as the x-ray source for transient diffraction.
\end{abstract}

\section{Introduction}

As vital tool in the study of matter with periodic and quasi-periodic structure, $\mathrm{x}$-ray diffraction has long involved some degree of temporal resolution ${ }^{1}$. Only in about the last 25 years, however, have $\mathrm{x}$-ray sources been powerful enough to resolve some of the most interesting physical phenomena such solid phase transitions, and shock dynamics ${ }^{2,3}$. In addition, the availability of powerful x-ray sources has fostered the need for methods of controlling and sampling the x-ray signals. An important review of transient diffraction ( both x-ray and neutron) has recently appeared ${ }^{4}$.

Laser-plasmas (LPs) have a number of characteristics that make them very valuable as $\mathrm{x}$-ray sources in time resolved diffraction experiments:

- a wide range of pulse durations from a few picoseconds (or less) ${ }^{5}$ to hundreds of nanoseconds.

- very bright $\mathrm{x}$-ray sources can be generated with source sizes as small as a few microns

- LP x-ray sources can be accurately synchronized with other events that can be driven, triggered, or otherwise stimulated by the laser light ${ }^{6}$.

The latter characteristic, is particularly important with respect to materials science applications. In this work, experiments frequently utilize laser-driven shock waves to measure properties such as the equation of state of a material, the generation of dislocations etc. Laser-based transient diffraction experiments can probe the state of shocked crystalline material by creating both the shock wave and the $\mathrm{x}$-ray source with the same high power laser. In addition to the synchronization between shock generation and the $\mathrm{x}$-ray source, laser-based experiments have several other advantages in materials work:

- the shock pressure can be accurately controlled and shaped through control of the laser temporal profile 
- with a single system, experiments can span a very wide range of pressures from 10 of kilobars to $100 \mathrm{Mbar}$ or more.

- laser experiments can have a very high data rate resulting in good assessment of accuracy and systematic trends.

In the present paper, we will emphasize the use of LP x-ray sources but considerable progress in transient diffraction has been made using e-beam ${ }^{7}$ and other sources. A review of the use of LP $\mathrm{x}$-ray sources is given in the aforementioned book on transient diffraction ${ }^{8}$. There is a complementarity between the 2 major applications of transient diffraction ( materials science and x-ray optics) that will be discussed in this review. Important materials effects such as the generation of dislocations can be studied by the alteration of $x$-ray diffraction ${ }^{9}$. Conversely materials effects such shock propagation, electrostriction etc. can be used control, modulate etc. $x$-rays in an electro-optic sense. These 2 aspects of transient diffraction in laser-based experiments have been recognized for some time ${ }^{10}$ but improvements in available laser systems have made progress especially rapid in the last 10 years.

We will first discuss some of the basic aspects of $\mathrm{x}$-ray diffraction and scattering. These will then be used as background for a discussion of the effects of various materials phenomena on the diffraction process. Current work on the application of transient diffraction ( using LP x-ray sources) to materials problems will be described. In much of this work, laser generated shock waves varying in pressure from a few hundred kilobars to several megabars are driven into materials having a fairly high degree of crystalline perfection. Diffraction is observed from a number of planes and can be used to make absolute, dynamic measurements of strain, probe the onset of plastic flow ${ }^{11}$, observe the onset of phase changes and other important properties. Finally we will describe some of the potential of and limitations on the use of transient diffraction for the modulation and control of $x$-rays.

\section{Basic X-ray Optics Involved in Transient Diffraction}

$\mathrm{X}$-ray propagation in materials differs considerably from optical phenomena. A dispersion calculation is similar to that in the optical regime but the shift to higher energies involves deeper resonances of the atoms until all or most of the resonances are exhausted. This leads to drastically different values of the refractive index ${ }^{12}$. For $x$-rays of moderate energies ( $a$ few keV and greater) the index is less than unity. This has a number of important implications for $\mathrm{x}$-ray optics. For example, the Fresnel reflection equations show that for such values of index, ordinary reflection can occur only at very shallow grazing angles. The small departure of the index from unity implies that no lenses of significant power are possible and only reflecting elements can be considered.

Media with periodic structure on the atomic or near-atomic scale such as natural crystals or layered synthetic multilayers (LSM) reflect $\mathrm{x}$-rays at angles more reminiscent of optical phenomena. The basics nature of propagation is, however, very different from that described by the Fresnel equations. The basic problem is to describe the propagation of electromagnetic radiation in a medium with periodically varying index of refraction. The general theoretical construct that has evolved for this problem is the dynamical theory of $x$-ray diffraction ${ }^{13,14,15}$.

Simple identification of diffracted peaks (obeying Bragg's law) from various planes in a solid can be accomplished by a simplified form of diffraction theory known as kinematical. In general, it can be said that this theory predicts to a good approximation the directional characteristics of the x-ray field and gives a rough approximation of the intensity. Kinematical theory does not take into proper account the effects of multiple reflections or of absorption. For work with large single crystals (which 
are the most common in transient diffraction work) and for the description of complicated disruptions of the lattice the more general dynamical theory is required. Taking into account the multiple reflections that occur in a large single crystal approximates the operation of an interferometer. In fact, the earliest and simplest development of the dynamical theory was closely analogous to the analysis of multilayer optical films ${ }^{16}$. In the limited space available here, we will give a brief description of this simple (Darwin) description of dynamical theory and indicate the extensions to more general theories.

Darwin's solution of the dynamical diffraction problem involves 3 basic steps.

1) First, the Fresnel diffraction problem for a plane populated with scattering centers (atoms) is solved. This gives an effective ( complex) reflection and transmission coefficient for x-rays incident on the atomic planes at a particular scattering angle $\theta$. This coefficient can be written:

$$
\mathrm{r}=\frac{\mathrm{e}^{2}}{\mathrm{mc}^{2}} \mathrm{M} \frac{\lambda \mathrm{f}(2 \theta)}{\operatorname{Sin}(\theta)}
$$

where $\mathrm{e}^{2} / \mathrm{mc}^{2}$ is the classical electron radius, $\mathrm{M}$ is the number of atoms/ unit area in the plane and $\mathrm{f}$ is atomic scattering factor at angle $\theta$. The transmission coefficient through a plane of atoms is $\left(1-\mathrm{h}+\mathrm{ir}_{0}\right)$ where $h$ is an absorption term representing the loss that a ray suffers in passing through a plane of atoms and $r_{0}$ is the value of $r$ at $\theta=0$.

2) The second step in the process is to use the reflection coefficient given in equation (1) and a corresponding transmission coefficient to formulate a series of difference equations that relate the electric field at a particular atomic plane to the fields one plane above and below. This step is illustrated in fig. 1 where $T_{i}$ and $R_{i}$ represent the transmission and reflection at any particular atomic plane

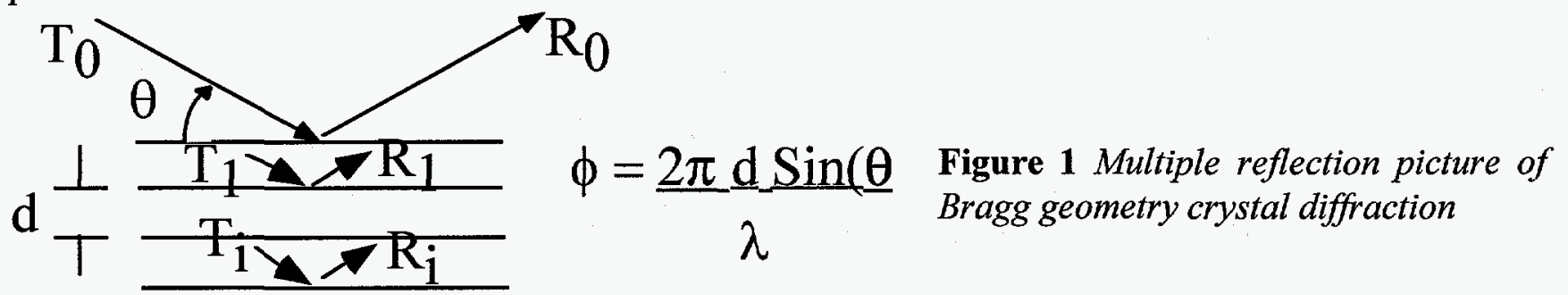

The resulting difference equations are:

$$
\begin{aligned}
& R_{i}=T_{i}(i r)+\left(1-h+i r_{0}\right) R_{i+1} e-i \phi \\
& T_{i+1}=\left(1-h+i r_{0}\right) T_{i} e^{-i \phi}+i r R_{i+1} e^{-2 i \phi}
\end{aligned}
$$

3 ) The third step in the process is to solve the difference equations under a variety of assumptions corresponding to particular crystals and their deviation from perfection. A matrix formulation ${ }^{10}$ (described below) of the Darwin equations has been used to obtain some of the interesting solutions. It is convenient to use the angle variable that represents the deviation from the exact Bragg condition:

$$
\Delta \theta=\frac{2 \pi}{\lambda}\left(\operatorname{Sin}\left(\theta-\operatorname{Sin} \theta_{B}\right)\right.
$$

The quantities $r, r_{0}$, and $\Delta \theta$ are small quantities (typically of the order $10^{-5}-10^{-4}$ ). Making some approximations for the phase factors we can write the following matrix equation:

$$
\left[\begin{array}{l}
T_{i} \\
R_{i}
\end{array}\right]=M\left[\begin{array}{c}
T_{i+1} \\
R_{i+1}
\end{array}\right]
$$


where $\mathbf{M}=\left[\begin{array}{cc}1 & -\left[2\left(\mathrm{rr}_{0}+\mathrm{r} \Delta \theta\right)+\mathrm{ir}\right] \\ \text { (ir) } & 1-\mathrm{h}-\mathrm{i}(2 \Delta \theta)\end{array}\right]$

Quantities such as the incident and reflected field amplitudes can now be expressed in terms of the matrix equations. For example in a crystal or multilayer with $n$ layers the effective reflectivity could be expressed as

$R=\frac{C^{*} C}{A^{*} A}$ where $C$ and $A$ are elements in the matrix $(M)^{\mathbf{n}}=\left[\begin{array}{ll}A & B \\ C & D\end{array}\right]$

As a simple example of the Darwin equations in matrix form we show in figure 2 the intensity reflection coefficient as a function of the number of lattice planes for the specific case or the $\langle 200\rangle$. reflection planes in $\mathrm{NaCl}$. This illustrates that under perfect satisfaction of the Bragg condition the process of diffraction can be quite efficient when the effective number of reflections is large (analogous to a large finesse in an optical interferometer).

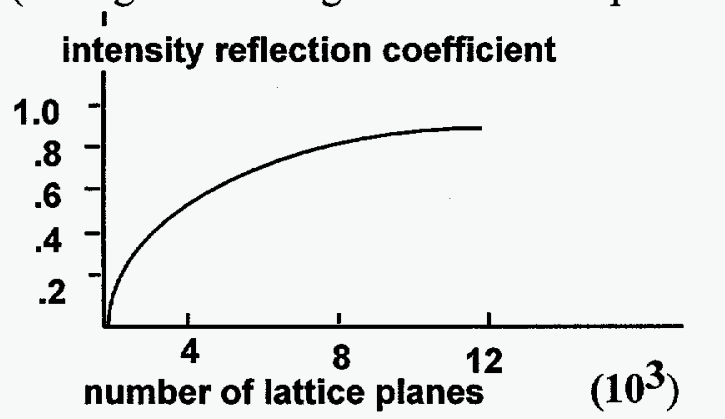

Figure 2 Numerical calculation of the $x$-ray reflection coefficient as a function of the number of lattice planes in the crystal

The Darwin equations can also be the starting point for expressing the effect of crystal distortion, imperfection etc. ( that can be caused by shock waves for example) on the diffraction process. For example as distortion in the lattice reduces the number of effective reflections (figure 2) the diffracted intensity will be correspondingly reduced. A generalization of these equations in the presence of lattice distortion has been presented in the work of Takagi ${ }^{17}$ and Taupin. The Takagi-Taupin equations have been solved under a variety of assumptions the most important being the restriction of spatially slowly varying strain. Since the matrix formulation of the multiple reflections equations was first presented ${ }^{10}$, similar formulations have been applied to the calculation of the reflection from layered synthetic multilayers ${ }^{18}$.

An alternate statement of the dynamical theory is often referred to as the Ewald-Von Laue formulation. The starting point for this picture is the expansion of the dielectric constant ( or alternately the crystalline structure factor) as a fourier series with the crystal reciprocal lattice vectors as the basis ${ }^{13}$. This leads to generalization of the familiar Ewald Sphere construction where allowed reflections are presented as a series of (usually hyperbolic) surfaces in reciprocal (spatial frequency) space.

Recently very convenient solutions to the dynamical equations for distorted lattices have been presented by Wark ${ }^{19}$ and Chukovskii ${ }^{20}$. Later in this paper we will show the use of the formulation by Wark in the prediction of diffraction from a lattice with a sinusoidal spatial perturbation. 


\section{Transient diffraction in materials science studies}

Flash x-ray diffraction was first applied to detailed ${ }^{\dagger}$ solid materials studies by Quentin Johnson in the early $1970 \mathrm{~s}^{2}$. He employed an e-beam flash $\mathrm{x}$-ray source to investigate materials under shock loading created by a high pressure gas gun. Most of his work was in the range of a few hundred kilobar pressure but some extended as high as $1 \mathrm{Mbar}$. In the early 1970s it was realized that high power laser systems could play a significant role in such work ${ }^{10}$ since they possessed the advantages pointed out in the first section of this paper. This work was also motivated by the possibility of using transient diffraction as a method for electro-optic control and switching of $x$-rays ${ }^{21,22,23}$.

There are 2 basic methods for using the laser to drive shock waves in solid systems. The first is direct optical ablation of the surface of a target. The ablation pressure created by this process can drive shock waves into solids over a very wide range of pressures. A rough feeling for the magnitudes involved can be obtained a simple model that regards the hot ablated material as the accelerating force in a "rocket" like calculation ${ }^{24}$. The shock pressure can be expressed as:

$$
\mathbf{P}=10 \lambda^{-2 / 3}\left(\mathbf{I} / 10^{14}\right)^{2 / 3}(\mathrm{Mbar})
$$

where I is the laser irradiance in watts $/ \mathrm{cm}^{2}$ and $\lambda=$ the laser wavelength (in microns).

As an example typical of the widely used $\mathrm{Nd}$ glass lasers, 30 joules of green laser energy in $1 \mathrm{~ns}$ focused to $6 \mathrm{~mm}$ will give an irradiance of $\sim 10^{11}$ watts $/ \mathrm{cm}^{2}$. Using eq. (4), this irradiance will produce a shock pressure of about $170 \mathrm{kbar}$. This is in the regime where many interesting solid-solid phase changes occur. Increasing this pressure by 1 to 2 orders of magnitude is well within the range of many current research laser systems. In the range of a few megabars shock melting of many important materials occurs ${ }^{25}$.

$\mathrm{X}$-ray radiation drive is the second major method of utilizing lasers for shock studies of solids. With this technique, laser radiation is introduced into a high $\mathrm{Z}$ metal cavity known as a hohlraum. The heating of the walls of this cavity produces a high temperature (typically $100-300 \mathrm{eV}$ ) nearly black body radiation field which can then be used to heat and accelerate materials and drive shocks. The shock pressure ${ }^{26}$ for a given hohlraum temperature is given by:

$$
\mathbf{P}_{\mathrm{a}} \sim 8 \mathbf{T}_{\mathbf{r}}{ }^{3.5} \text { (Mbar) }
$$

where the radiation temperature is in $10^{2} \mathrm{eV}$. This, for example, tells us that for $200 \mathrm{eV}$ hohlraum drive, ablation pressures of about $100 \mathrm{Mbar}$ can be generated. To produce these temperatures over a 1$2 \mathrm{~mm}$ sample about 15 kilojoules of laser energy are required - a level available on only a few lasers in the world. Fortunately a large number of important phenomena in shock studies of solids occur at pressures around $10 \mathrm{Mbar}$ and lower. Investigations of this region of pressure can be undertaken on laser systems with less than a kilojoule of energy.

The final requirement for utilizing lasers in materials studies is the generation of modest photon energy ( $2-10 \mathrm{keV}$ ) x-rays for probing, using techniques such as transient diffraction. The details of the use of laser plasmas as a source for transient diffraction and other methods is given by Hauer and $\mathrm{Kyrala}^{8}$ in the recent volume on transient diffraction ${ }^{4}$. In summary, current systems can provide the following characteristics:

- photon energies from sub kilovolt to $>10$ kilovolts

\footnotetext{
${ }^{\dagger}$ Earlier work of a more preliminary nature was done by Al'tshuler in Russia (in 1941) and others
} 
- pulse durations from a few picoseconds to 10 of nanoseconds

- source sizes from about 10 microns about $1 \mathrm{~mm}$.

Such a range of source parameters provides a great flexibility in diagnostics of materials phenomena.

We will now give a few examples of current work utilizing lasers for shock materials studies. One interesting issue that can be addressed by transient diffraction is the mechanism and rate of dislocation generation behind a shock front ${ }^{9}$. At high strain rates $\left(\sim 10^{6}\right.$ and greater) these mechanisms are poorly understood. Direct measurements of both the elastic and plastic components of strain would be very valuable in helping to clarify this phenomena. Transient diffraction is one possible method. Another interesting issue is the study of the details of melting and recrystallization of materials. For example studies of alkali halide crystals ${ }^{27}$ show that an important material such as $\mathrm{LiF}$ melts at about $1 \mathrm{Mbar}$ a range quite easily addressed by laser experiments.

In figure 3 we show an experiment that was performed on the Trident laser system at Los Alamos National Laboratory. In this test, shock waves were driven into various single crystals using the direct illumination method. The shock excitation beam used between 20 and $100 \mathrm{~J}$ of laser energy in $1 \mathrm{~ns}$ pulses. The irradiance level was about $10^{12} \mathrm{~W} / \mathrm{cm}^{2}$, driving a modest-strength shock into the crystal. Another arm of the laser system is used to create an $\mathrm{x}$-ray probe beam with photon energies in the $3-7 \mathrm{keV}$ range. The $\mathrm{x}$-ray probe source can be operated in 2 basic temporal modes. In the short pulse "flash" mode, a single short laser pulse creates a short x-ray burst in the range of 10 to a few hundred picoseconds ( depending on the phenomena being studied). This x-ray burst is then used to diffract or scatter off of sample through which the shock wave is propagating. In the other mode, a relatively long pulse ( typically a few nanoseconds) $\mathrm{x}$-ray probe is created and temporal resolution is obtained with an X-ray streak camera or gated framing detector ${ }^{8}$ This mode enables one to investigate nearly the full temporal history of the shock propagation event. In the present experiment, a laser pulse of about $30 \mathrm{~J}$ energy and a pulse duration of $100 \mathrm{ps}$ (at a wavelength of $0.53 \mathrm{~mm}$ ) was tightly focused to a small spot on a target containing $\mathrm{Cl}$. This beam created a bright source of $\mathrm{Cl}\left(1 \mathrm{~s}^{2}-1 \mathrm{~s} 2 \mathrm{p}\right)$ line radiation at $4.4 \AA$, which was diffracted from the [111] planes of a Si crystal.

An additional feature in this experiment was the introduction of a spatial modulation on the shock driving beam by creation of an optical interference pattern. This resulted in the propagation of a spatially rippled shock or stress wave into the sample. By delaying the x-ray diffraction source pulse with respect to the shock-inducing beam, the evolution of the imprint of the rippled shock can be studied. In addition to the general evolution, a quantitative measurement of the strain level can be made.

At an irradiance level of slightly less than $10^{12} \mathrm{~W} / \mathrm{cm}^{2}$, a shock wave of a few hundred kilobars is generated. By changing the irradiance levels, the pressure can easily be changed from a few tens of kilobars to several tens of megabars. This spans a number of interesting materials transitions, including the elastic-plastic transition and melting. In this particular experiment, the introduction of a modulation on the laser beam as shown in Figure 3 had two motivations. First, the propagation of a structured shock through materials is a classical problem ${ }^{28}$ that sheds light on the properties of materials near 


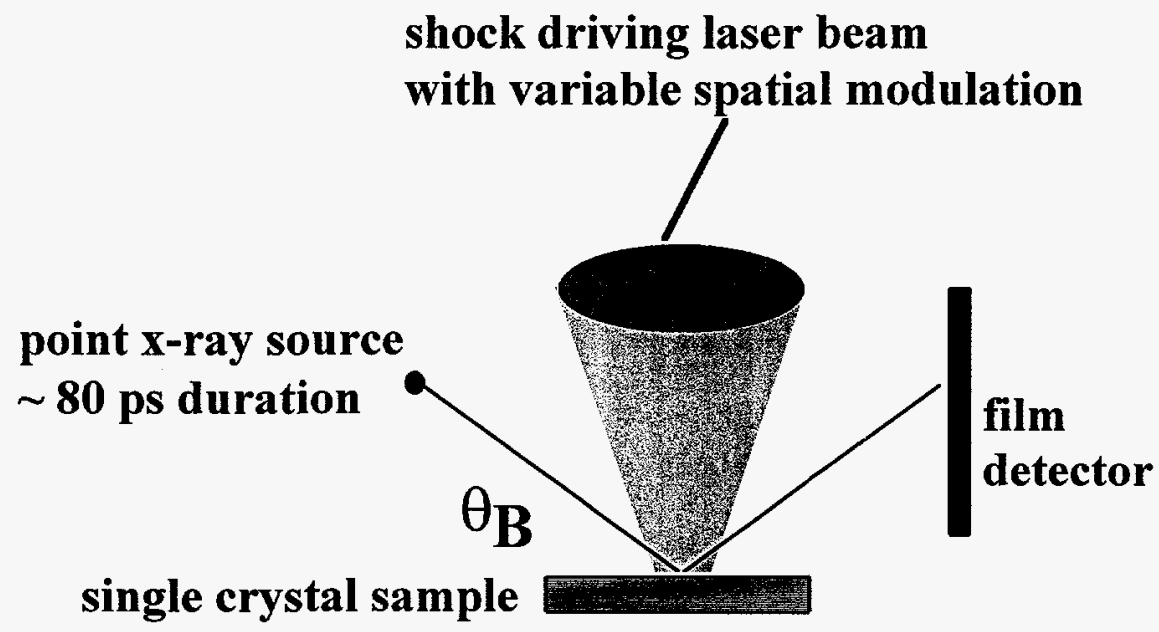

Figure 3. Configuration for the study of moderate-pressure shock/stress-wave propagation in crystalline material. An interference pattern modulation on the shock-driving laser beam is introduced (roughly orthogonal to the $x$-ray dispersion direction).

phase transitions. In figure 4, we show the results of 2 tests (at different shock pressures) using this transient diffraction configuration. The breakup of the diffraction at the higher pressure may be due to a phase transition or the onset of plastic flow. The shock pressures in the 2 experiments shown here were too low to have produced melting.

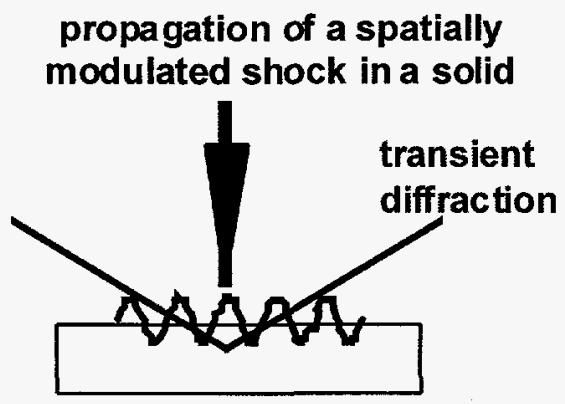

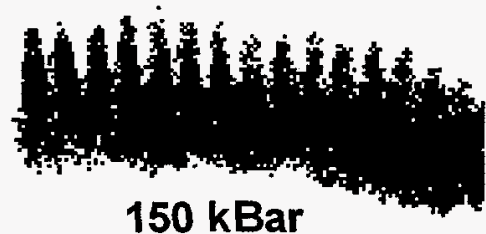

150 kBar

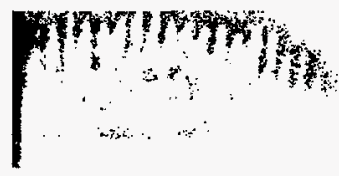

$>250 \mathrm{kBar}$

Figure 4. Transient $x$-ray diffraction from material with a spatially modulated shock propagating through it. Magnification in the 2 diffraction patterns is slightly different.

Some preliminary work has been done to model the x-ray diffraction signature of the propagation of these rippled shocks. The modeling begins with the use of a hydrodynamics code that simulates the laser absorption and the formation of the shock. The predicted values of strain in the solid are then used as input for the dynamical diffraction formulation developed by Wark. In figure 5 we show the results of one such simulation.

spatial dimensions on the film detector

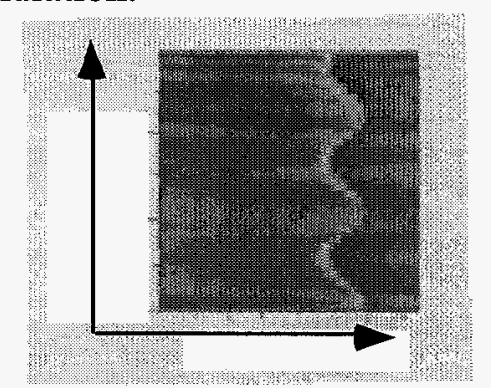

Figure 5. Simulation of the transient $x$-ray diffraction from a spatially rippled shock propagating in a silicon single crystal. The image can be used to directly measure the real time strain levels in the crystal

Recently a very useful analytical analysis of the propagation of rippled shocks has appeared in the literature $^{29}$. The second motivation for the study of the propagation of modulated shocks is the 
"imprint problem" encountered in direct drive laser fusion ${ }^{30}$. Imperfections in a laser beam irradiating a solid target create ( or "imprint") a rippled shock wave which can later be amplified by hydrodynamic instabilities creating a degraded implosion. Transient diffraction can be used to study the early phases of the imprint process.

In figure 6, we show an example of a shock materials experiment utilizing x-ray hohlraum drive. This test was performed on the Nova laser system at Lawrence Livermore Laboratory. Smooth uniform drive is achieved in these experiments through the natural symmetrization that occurs in the quasi- black body radiation environment in the hohlraum. To achieve the best uniformity using the direct drive method special spatial smoothing techniques such as the introduction of special phase plates. are required.

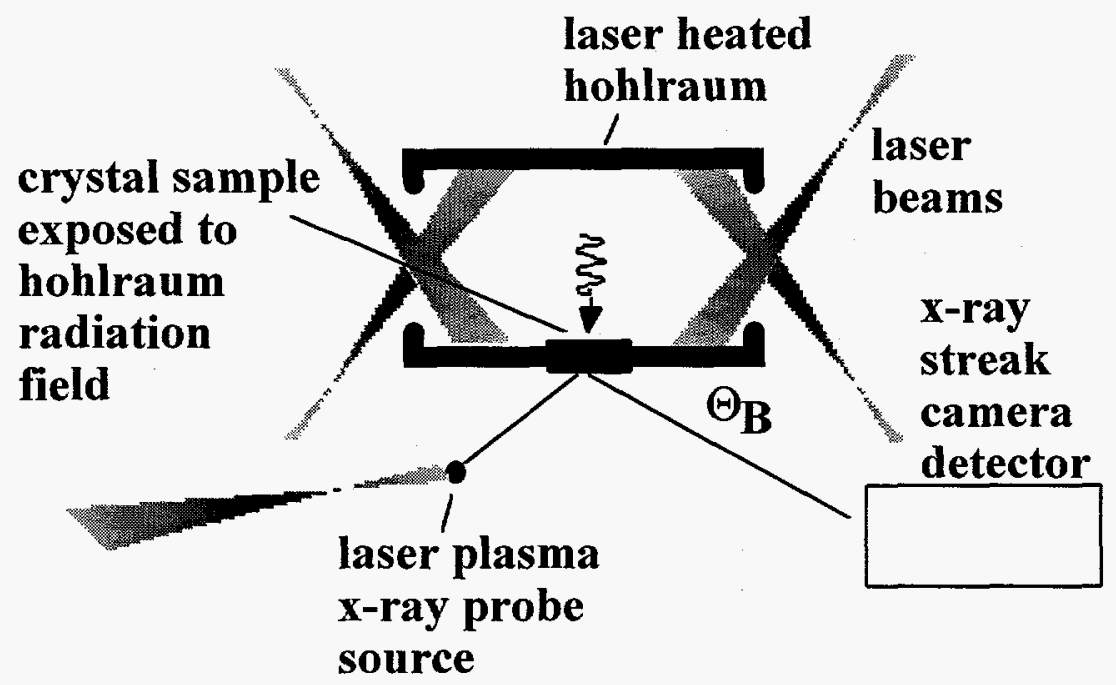

Figure 6. Configuration for transient diffraction experiments utilizing $x$-ray (hohlraum) drive for the excitation of the shock. The $x$-ray streak detection allows a continuous record of the diffraction during much of the shock propagation in the crystal. Internal shields in the hohlraum are not shown.

In figure 7 the results of a transient diffraction experiments using hohlraum drive are shown. About 5 $\mathrm{KJ}$ of laser radiation was used to heat the hohlraum to about $40 \mathrm{eV}$ driving a shock of over a megabar into the silicon crystal.

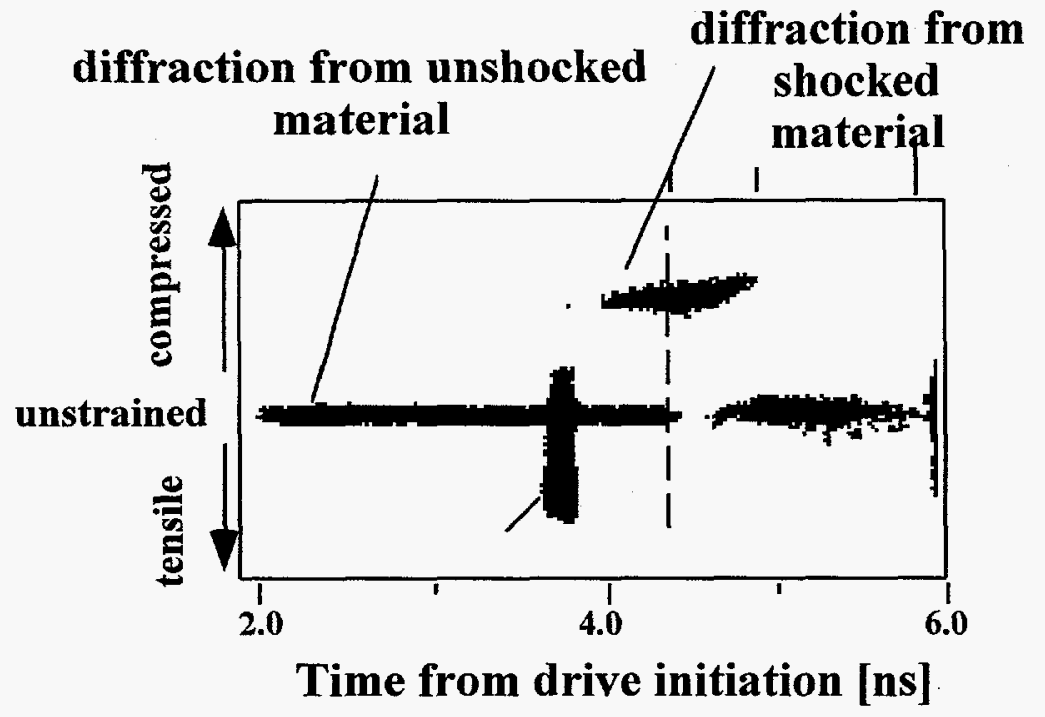

Figure 7.

time resolved diffraction from $x$-ray driven shock in a silicon single crystal. Strains as large as $15 \%$ over a nanosecond have been demonstrated by such a technique. $5 \mathrm{KJ}$ in 2 ns of laser energy were used to heat the hohlraum The disappearance of diffraction may indicate the onset of melt. 
In order to study both the elastic and plastic components of strain, it is necessary to measure the diffraction from more than one set of planes simultaneously. A convenient geometry for this is illustrated in figure 8 where orthogonal diffraction from both "Bragg" and "Laue" geometry is observed. Initial work utilizing this geometry was performed by Whitlock and Wark ${ }^{11}$. In order to obtain quantitative determination of plastic behavior, however, it will be necessary to obtain temporal resolution of both the Bragg and Laue diffracted radiation. A preliminary experimental result showing such a measurement from a directly shocked Lif crystal is shown in figure 8.

"Laue" diffraction

from planes perpendicular to the entrance surface

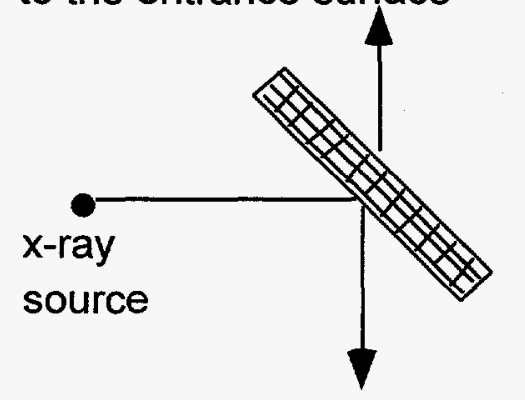

"Bragg" diffraction from planes parallel to the entrance surface

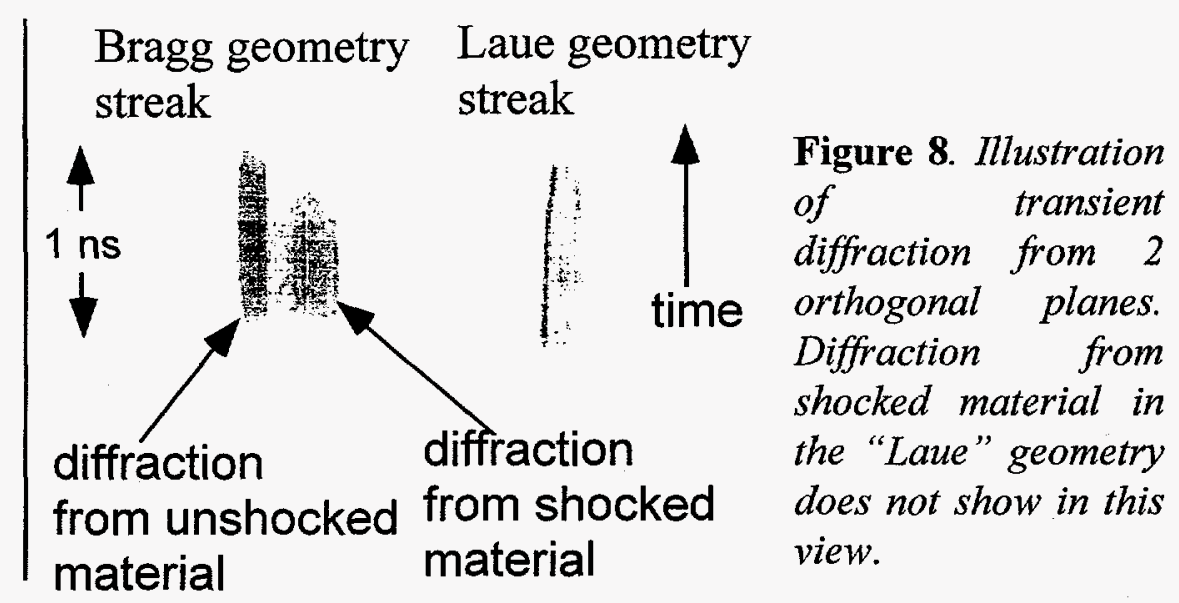




\section{X-ray Optical Effects Utilizing Transient Diffraction and Scattering}

The use of transient diffraction as a method for the control of x-ray propagation has been under investigation for over 20 years ${ }^{10}$. In recent years the advent of lasers with ultrashort pulses and very high irradiance have significantly increased progress in this work ${ }^{31}$

Typically fast ( subnanosecond) switching or shuttering devices, in the optical regime, operate through use of some form of the electro-optic effect. Electro-optic effects at x-ray wavelengths ( changing the $\mathrm{x}$-ray polarizibility of a material) is more difficult to observe. On the other hand, x-ray wave propagation in a solid can be markedly affected by changing the relative positions of atomic scatterers within the material. This is dramatically illustrated by the very narrow angular acceptance of Bragg reflections of monochromatic x-rays from a perfect crystal. Very small perturbations of the effective atomic spacing in crystal can result in large changes in $\mathrm{x}$-ray reflection.

A dispersion calculation for the $\mathrm{x}$-ray refractive index is similar to that in the optical regime but with some modifications. When the x-ray wavelength becomes comparable to the atomic dimensions ( the regime of most interest in transient diffraction) interference effects between the various electrons in the atom must be taken into account. Since we are typically dealing the case where the photon energy is greater than all or most of the atomic resonances, the scattering process is quasi-free (i.e. Thomson) but the distribution of electrons within the atom is obtained from quantum mechanical calculations. The electrons start to participate more or less equally in the scattering of x-rays. The detailed calculations actually show the scattering cross sections to be more heavily weighted toward the inner shell electrons. Thus in order to produce electro-optic effects directly, externally applied fields must perturb the whole electronic cloud. This in turn requires electric field strengths of the order that would be required to ionize all or most of the electrons. Such field strengths have only been available in very recent years with the advent of ultrashort pulse lasers. In essence the above description shows that it is quite difficult to affect the real part of the refractive index.

It is somewhat easier to affect the imaginary part of the index which is due to absorption ( due usually to the photoelectric effect). The time required to produce a high ionization state of a target can be quite fast. Suppose for example that $44 \AA$ (carbon $\mathrm{K}_{\alpha}$ were being used to probe a laser produced aluminum plasma. If the initial target consisted of a one micron foil, it would transmit only

$\mathrm{e}^{-8.1}$. When the aluminum is heated with a flux density of $10^{14} \mathrm{~W} / \mathrm{cm}^{2}$ or greater helium and hydrogenlike states are rapidly produced. Once these states are produced the transmission of the foil changes considerably. Since this method of probing was first proposed 20 years ago ${ }^{10}$ it has been used in several investigations to probe the ionization state of plasmas ${ }^{32}$.

The most effective way to control $x$-ray propagation is to alter the configuration of the electronic $\mathrm{X}$-ray scatters within a solid. Shock waves or acoustic phenomena propagate on a rather slow time scale (of the order of $10^{6} \mathrm{~cm} / \mathrm{sec}$ or less). A shock wave can still have an important effect on diffraction on a fast time scale. As is illustrated in fig. 2 the Bragg reflection is quite efficient and can reflect a large portion of the input beam after passing through only 1000 or so atomic layers. This results in so called "extinction" depths of only a few microns in typical cases. A relatively powerful shock wave can penetrate this depth in a few hundred picoseconds. For faster interaction, an incident electromagnetic wave must interact directly with the "optical" branch of lattice vibrations. Analysis of the possible methods of exciting appropriate lattice vibrations ${ }^{10}$ showed that it was highly likely that significant diffraction effects could be observed on time scales faster than $10 \mathrm{ps}$. For example, in figure 9 we show an experimentally determined phonon curve for a simple cubic lattice. The optical branches 
of the dispersion curves clearly have frequencies that could produce motion on a picosecond time scale. The terminology of "optical branch" arises from the fact that adjacent atoms vibrate in an opposite sense, so that if the binding energy is such that charge separation is caused by this mode , an electromagnetic wave could couple energy in directly. The frequencies in this case correspond to the far infrared.

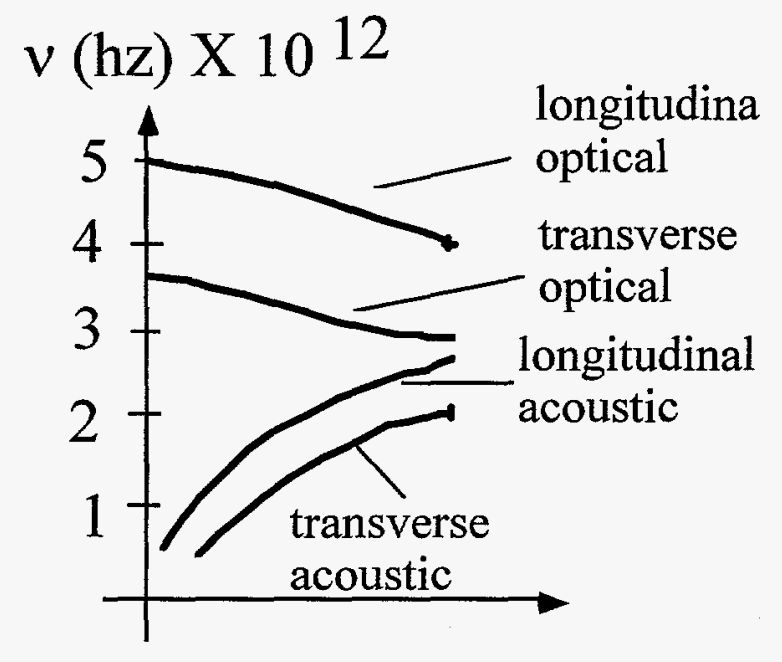

Figure 9. Typical phonon dispersion curve for a simple diatomic cubic lattice.

In recent picosecond diffraction experiments, it is a question as to whether coupling to the lattice modes has caused simple heating. If this is the case, the reduction in diffracted intensity should be similar to the classical Debye- Waller factor ${ }^{12}$. If this is the mechanism as is pointed out in ref. 10 it will be difficult to achieve large changes in x-ray signal. This would be a detriment for use in electro-optic control applications but still should provide very valuable information on solid dynamics.

At the time of the early analysis ${ }^{10}$ lasers were not capable of producing the field strengths necessary for many of these effects. Now that fields generated by very high irradiance lasers are available, it should be possible to observe many picosecond diffraction effects such as those reported recently ${ }^{31}$.

\section{References}

${ }^{1}$ See for example E. P. Bertin Principles and Practice of X-ray Spectrometric Analsis, Plenum, New York 1975. ; Al'tshuler and colleagues in Russia produced "flash" $\mathrm{x}$-ray diffraction patterns as early as 1941.

${ }^{2}$ Q. Johnson, A. Mitchell, R. N. Keeler and L. Evans, Phys. Rev. Lett. 25, 1099 (1970).

3 J. S. Wark, R. Whitlock, A. Hauer, J. Swain, and P. Solone, "Subnanosecond x-ray diffraction from laser-shocked crystals," Phys. Rev. B, 40 (8), 5705 (1989).

4 Time Resolved Diffraction edited by J.R. Helliwell and P.M. Rentzepis published Oxford Clarendon Press 1997,

${ }^{5}$ M.M.Murnane, et al. (1994), Appl. Phys. B 58, 261.

${ }^{6}$ A.A.Hauer, et al. (1989), Current new applications of laser plasmas, in Laser Induced Plasmas and Applications, Radziemski, L.J., and Cremers, D.A., Eds., Marcel Dekker, New York and Basel, 385.

${ }^{7}$ I.V., Tomov , P. Chen, and P. Rentzepis (1995), Nanosecond hard x-ray source for time resolved xray diffraction studies, Rev. Sci. Instrum. 66(11), 5214.

${ }^{8}$ A.A. Hauer, G. A. Kyrala, Laser-Plasma X-ray Emission: Its Creation, Diagnosis and Application in Transient Diffraction in Time Resolved Diffraction edited by J.R. Helliwell and P.M. Rentzepis published Oxford Clarendon Press 1997,

${ }^{9}$ E. Zaretsky, J. Appl. Phys. 78(6), 3740, 1995. 
${ }^{10}$ A.A. Hauer, A Study of the Interaction of X-rays and Acoustic, Stress and Shock Waves in Solids Los Alamos National Laboratory Report 97-1698 ( Available from US NTIS or Los Alamos National Lab.) ; first published by University Microfilms -1976.

${ }^{11}$ R. R.Whitlock, J.S. Wark, Phys. Rev.B. 52, 8 (1995).

12 see for example A. H. Compton and S. K. Allison X-rays in Theory and Practice, D.Van Nostrand, Princeton, 1954.

${ }^{13}$ W.H. Zachariasen Theory of X-ray Diffraction In Crystals, Dover, (1967).

${ }^{14}$ B.W. Batterman and H. Cole "Dynamical Diffraction of X-rays by Perfect Crystals", Rev. of Mod. Phys. 36(3), 1964.

15 L. Brilliouin, Wave Propagation in Perioic Structures, Dover, (1946).

${ }^{16}$ C.G Darwin, Phil. Mag. 27, 315 (1914), and Phil. Mag. 43,800 (1922).

17 S. Takagi " A Dynamical Theory of Diffaction for Distorted Crystal" J. of Phys. Soc. of Japan 26(5), 1239, (1969).

${ }_{18}$ P. Lee, Opt. Comm. 37(3), 159, 1981

19 J. S. Wark et al., J Appl. Phys. 68, 4531 (1990).

${ }^{20}$ F. N. Chukovskii, C. Malgrange, J. Gronkowski, Acta. Cryst. A52, 47,1996

${ }^{21}$ Hauer, A.A., and Burns, S. (1975), Appl. Phys. Lett. 27(10), 524.

${ }^{22}$ A. Hauer, U.S. Patent 3,991,309 "Methods for the Control and Analysis of X-rays" -1976.

${ }^{23}$ Wark, J.S., Hauer, A.A , and Kilkenny, J.D. (1986), Rev. Sci. Instrum. 57, 2168.

${ }^{24}$ J.J. Duderstadt, G. Moses, Inertial Confinement Fusion, Wiley, 1981.

${ }^{25}$ G.E. Duvall, R.A. Graham, Rev. Mod. Phys. 49(3), 523, 1977.

${ }^{26}$ J. Kilkenny, "Inertial Confinement Fusion" in Laser Plasma Interactions-5 Published by Scottish Universities Summer School, Ed. M. B. Hooper, 1995.

${ }^{27}$ R. Boehler, M. Ross, D. Boercker, Phys. Rev. Lett. 78(24), 4589, 1997.

${ }^{28}$ A. D.Sakharov, et al. (1965), "Experimental investigation of the stability of shock waves and the mechanical properties of substances at high pressures and temperature", Sov. Phys. Dok. 9(13), 1091.

${ }^{29}$ R. Ishizaki, K. Nishihara, Phys. Rev. Lett. 78(10), $1920,1997$.

${ }^{30}$ D. Kalantar et.al. Phys. Rev. Lett. 76(19), 3574,1996

${ }^{31}$ J. Larson et. al. Opt. Lett. 22(13), 1012,1997. ; see also M. Ben-Num, K. R. Wilson, paper 3157-15 in this conference.

${ }^{32}$ D. Casperson et. al. J. App. Phys. 74((6), 3707,1993.

\section{Acknowledgments}

The authors would like to express their appreciation for the technical assistance that was vital in obtaining much of the data described in this paper. This help came from the operating teams at the Trident and Nova laser systems and from the fine technician teams at Los Alamos and LLNL. In addition the materials science / target fabrication teams at Los Alamos and Livermore supplied the precision targets necessary for this work.

Much of the work described here was work performed under the auspices of the U.S. D.O.E. under contract no. W-7405ENG-36. 